\title{
Experimental study of kidney protective effects of sanhuangshaoshangling on severe burn rats in shock stage
}

\author{
Guolin $\mathrm{Hu}^{1}$, Lingfeng Wang ${ }^{* 2}$, Bo Feng ${ }^{2}$ \\ ${ }^{1}$ College of Life Science, Inner Mongolia University, Hohhot, Inner Mongolia, China \\ ${ }^{2}$ Baogang Hospital, Inner Mongolia, China
}

Received: June 18, 2016

DOI: $10.14725 /$ dcc.v3n3p1

\author{
Accepted: July 21, 2016 \\ Online Published: September 10, 2016 \\ URL: http://dx.doi.org/10.14725/dcc.v3n3p1
}

\begin{abstract}
Objective: To observe the protective effects and mechanisms of topical Chinese medicine on kidneys of severe burns shock rats.

Methods: Blood urea nitrogen (Bun), creatinine (Cr), myeloperoxidase (MPO), tumor necrosis factor- $\alpha$ (TNF- $\alpha$ ) and interleukin (IL-6), were checked by the time points of 8, 24, 48 hours. Blood tests, ELISA and other methods were used for these texts.

Results: Compared with the povidone-iodine group, the levels of Bun and $\mathrm{Cr}$ were declined apparently in the Sanhuang group, and the activity of MPO was decreased as well.

Conclusions: Sanhuangshaoshangling, taking ointment as matrix, can be externally applied to wounds. It contains some effective ingredients which can clear heat-toxicity and promote blood circulation for removing blood stasis, and prevents wounds from the stimulation of the air to nerve endings and the invasion of bacteria. It can also inhibit bacteria from parasitizing, growing, reproducing on the wound. Sanhuangshaoshangling helps blood circulation and facilitates the growth of tissues to inactivate the toxicity or reduce toxin by changing the structure of endotoxin after uptake into tissues. It can protect viscera by reducing the release of endotoxin, facilitating the excretion of endotoxin and weakening or eliminating the effects of TNF- $\alpha$ and IL-6.
\end{abstract}

Key Words: Sanhuangshaoshangling, Shock stage, MPO, TNF- $\alpha$, IL-6

Although traditional Chinese medicine for the treatment of shock (syncope and collapse) has already been documented, it is unable to be developed widely and intensively due to various factors, such as experimental methods, experimental techniques, pharmaceutical preparations etc. In recent years, the advances in the development of dosage form of traditional Chinese medicine have facilitated greatly a comprehensive and intense study in mechanisms of anti-shock prescriptions. Not only the effects on hemodynamics and hemorheology have been studied, but also anti-lipid peroxidation and protection of organ morphology etc. are being studied intensively.
According to science of traditional Chinese medicine, clinically, patients with renal insufficiency suffer from different degrees of "heat syndrome", interior retention of heat pathogen can be caused not only by overuse of pungent and heat food and abuse of hormones, but also endogenous or exogenous heat pathogen. Intense heat can damage the kidney and lead to hematuria, it can also make the kidney unable to govern storage and result in proteinuria. Heat pathogen can also be combined with dampness pathogen to form damp-heat syndrome, resulting in a longer course of renal insufficiency and many complications, therefore renal insufficiency can be treated by clearing heat and promoting

*Correspondence: Lingfeng Wang; E-mail: wlf7413@ vip.sina.com; Address: Baogang Hospital, Inner Mongolia, China. 
diuresis. Traditional Chinese medicine mainly intervenes in the acute kidney injury to protect the renal function by promoting blood circulation for removing blood stasis.

Sanhuangshaoshangling used in our hospital is made from scutellaria baicalensis, coptis chinensis, platycladus orientalis, lonicera japonica, bletilla striata and other ten more traditional Chinese herbs of a reasonable formula. It has an effect of heat-clearing and damp-drying. Meanwhile, it can also eliminate necrotic tissues and promote granulation, remove blood stasis for promoting tissue regeneration, promote blood circulation for relieving pains, as well as tonify Qi for promoting granulation. By reducing the release of vasoactive substances and mediators of inflammation, it can fight against oxidative stress injuries occurred in various cases and provide a protection for kidneys, lungs, intestines and other important organs.

\section{Materials and methods}

\subsection{The preparation of Sanhuangshaoshangling}

Sanhuangshaoshangling, whose formula is a cipher prescription for medical treatment in our hospital, is summarized from long-term clinical practices and clinically verified for a long time. This formula is of novelty, unique efficacy and safety. Sanhuangshaoshangling is made from scutellaria baicalensis, coptis chinensis, platycladus orientalis, lonicera japonica, bletilla striata and other ten more traditional Chinese herbs of a reasonable formula. Among those herbs, scutellaria baicalensis, coptis chinensis and platycladus orientalis are characterized by bitter-cold nature and flavor, with effects of heat-clearing and damp-drying, purging intense heat for detoxication and diminishing inflammation to excrete heat-toxin. Modern pharmacological studies have shown that berberine in coptis chinensis and platycladus orientalis has a strong inhibiting and sterilizing effect on Pseudomonas aeruginosa, Staphylococcus aureus and Escherichia coli. Chlorogenic acid in lonicera japonica has a strong inhibiting effect on Staphylococcus aureus and Escherichia coli. Bletilla striata has an inhibiting effect on Staphylococcus spp. Berberine also takes an analgesic action by relieving arrectores pilorum spasms. Angelica sinensis, bletilla striata and portulaca oleracea are of stypticity. Borneol has a function of anti-inflammation and relieving pain and swelling. With this combination of ingredients, Sanhuangshaoshangling has good therapeutic effects on burn wounds by sterilizing bacteria, diminishing inflammation, relieving pains, promoting granulation and facilitating wound healing. Therefore, the prescription source is reliable and conforms to the traditional Chinese medicine theory.

\subsection{Animal grouping and preparation of scald mod- els}

Healthy adult Wistar rats (provided by Inner Mongolia University) were randomly divided into 3 groups (control group, povidone-iodine group and Sanhuang group), each of which was subdivided into three groups according to different post-burn times ( 8 h, $24 \mathrm{~h}$ and $48 \mathrm{~h}$ ). Each group consisted of 6 rats of either gender, weighed 200-250 g. Rats in the control group were left intact; rats in scald model group kept fasting for $12 \mathrm{~h}$ before getting injured, and received intraperitoneal anesthesia by pentobarbital sodium with the mass fraction of $1 \%(30 \mathrm{mg} / \mathrm{kg})$. The rats were depilated on the back by a compound of barium sulfide, soap powders and talc powders in the proportion of 1: 1: 2 after hair-removal (by electric hair clipper) on the back. After 24 h-raising, the depilated areas of the rats were placed under hot water at $80^{\circ} \mathrm{C}$ for $15 \mathrm{~s}$, resulting in deep second-degree scald with $30 \%$ TBSA (confirmed by pathological section). Ringer's solution $(40 \mathrm{ml} / \mathrm{kg}$ ) was injected into the abdominal cavity after being scalded for fluid resuscitation. The rats were fed separately and had free access to water. The rats in the control group were treated in the same way without being burned.

\subsection{Sampling and specimen processing}

$2 \mathrm{ml}$ of blood was taken from abdominal aorta in each Wistar rat after anesthesia on different time points. Biochemical tests were carried out in order to detect blood urea nitrogen (Bun), creatinine (Cr), tumor necrosis factor- $\alpha$ (TNF- $\alpha)$ and interleukin (IL-6).

\subsubsection{Measurement of renal function}

Bun and Cr were measured by use of automatic biochemistry analyzer.

\subsubsection{Tissue specimens}

Renal tissues were taken from each rat in the control group. Other rats were also required of renal tissues after blood sampling on different time points. These tissues were fixed in $10 \%$ neutral formaldehyde for $24 \mathrm{~h}$. The fixed tissues were rinsed out, dehydrated by use of graded ethanol, vitrified with dimethylbenzene, immersed in petrolatum and embedded to make the preparation of paraffin blocks and sections for pathological examination and ELISA.

\subsubsection{Measurements of MPO}

MPO of renal tissues in rats was measured according to the instructions of ELISA. 


\subsubsection{Measurements of TNF- $\alpha$ and IL-6 in serums}

Anesthesia was carried out for healthy rats and burned rats on different time points. Under the aseptic condition, venous blood was taken from hearts and injected into clean tubes for auto-agglutination at $4^{\circ} \mathrm{C}$. The serum was taken out and refrigerated at $-20^{\circ} \mathrm{C}$ for the detection of TNF- $\alpha$ and IL-6. Radio immunoassay was applied to the measurement of TNF- $\alpha$ and IL- 6 concentrations, kits were provided by Beijing Institute of East Asian Immunology Technology and used as instructed.

\subsection{Statistical treatment}

SPSS11.0 was applied to statistical treatment. All of the data were represented by $\bar{x} \pm \mathrm{s}$. The difference $p<.05$ was of statistical significance. Groups of the same time point and different groups were tested by use of $t$-test, which was appropriate to the comparison between specimen means in completely random design.

\section{Results}

\subsection{Measurement results of renal function}

Compared with the control group, the values of Bun and $\mathrm{Cr}$ in plasma were obviously increased in the Sanhuang group and the povidone-iodine group on different time points $(p<.05)$, the values of Bun and $\mathrm{Cr}$ in plasma were lower in the Sanhuang group than those in the povidone-iodine group $(p<.05$, see Table 1$)$.

Table 1: Changes in renal function in rats of each group on different time points $(\bar{x} \pm \mathrm{s})$

\begin{tabular}{|c|c|c|c|c|c|c|c|}
\hline \multirow{2}{*}{ Group } & \multirow{2}{*}{$\begin{array}{l}\text { Number } \\
\text { of Rats }\end{array}$} & \multicolumn{3}{|c|}{ Bun/(mmol/L) } & \multicolumn{3}{|c|}{$\mathrm{Cr} /(\mu \mathrm{mol} / \mathrm{L})$} \\
\hline & & $8 \mathrm{~h}$ & $24 \mathrm{~h}$ & $48 \mathrm{~h}$ & $8 \mathrm{~h}$ & $24 \mathrm{~h}$ & $48 \mathrm{~h}$ \\
\hline Control group & 6 & $5.1 \pm 1.4$ & $5.3 \pm 1.4$ & $5.2 \pm 1.6$ & $37.4 \pm 2.1$ & $36.5 \pm 3.2$ & $38.2 \pm 3.1$ \\
\hline $\begin{array}{l}\text { Povidone-iodine } \\
\text { group }\end{array}$ & 6 & $15.6 \pm 2.1^{*}$ & $17.2 \pm 2.4^{*}$ & $24.3 \pm 2.1^{*}$ & $62.3 \pm 2.3^{*}$ & $75.0 \pm 3.4^{*}$ & $91.3 \pm 2.6^{*}$ \\
\hline Sanhuang group & 6 & $12.3 \pm 2.3^{* \#}$ & $14.6 \pm 2.1^{\text {*\# }}$ & $20.6 \pm 2.8^{* \#}$ & $48.5 \pm 2.6^{* \#}$ & $64.2 \pm 2.9^{* \#}$ & $72.5 \pm 2.8^{* \#}$ \\
\hline
\end{tabular}

Note. In comparison with the control group, ${ }^{*} p<.05$; In comparison with the povidone-iodine group, ${ }^{\#} p<.05$

\subsection{Changes in the activity of MPO in renal tissues}

Compared with the control group, the activity of MPO in the povidone-iodine group was obviously increased $(p<.05)$; Compared with the povidone-iodine group, the activity of MPO in the Sanhuang group was obviously decreased $(p<$ .05 ), but it was still higher than that in the control group (see Figure 1).

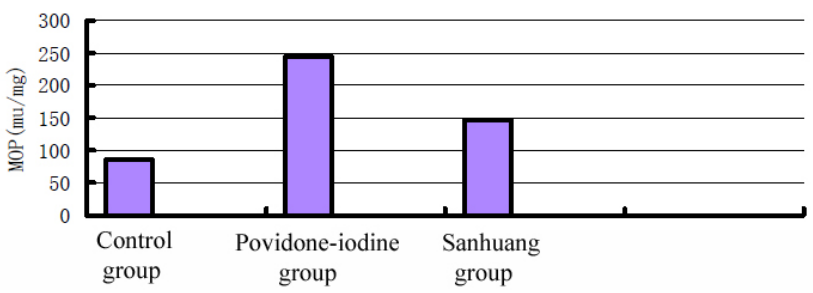

Figure 1: Changes in the activity of MPO in renal tissues

\subsection{Histopathological changes}

Under the light microscope, it could be observed that pathological lesions in renal tissues were definite in the povidoneiodine group with cellular swelling and necrosis. A large number of red blood cells and white blood cells were infiltrated into intercellular substances. The degree of pathological lesions in the Sanhuang group was less severe than that in the povidone-iodine group.

\subsection{Changes in the level of serum TNF- $\alpha$}

The levels of TNF- $\alpha$ in the povidone-iodine group and the Sanhuang group began to increase respectively on the time point of $8 \mathrm{~h}$ after burn injury, and no obvious difference in TNF- $\alpha$ concentration could be seen in these two groups on the time point of $24 \mathrm{~h}$. On the time point of $48 \mathrm{~h}$, the level of TNF- $\alpha$ in the Sanhuang group was obviously lower than that in the povidone-iodine group (see Figure 2).

\subsection{Changes in the level of serum IL-6}

The level of IL-6 in the Sanhuang group began to increase apparently on the time point of $8 \mathrm{~h}$ after burn injury, and the level in the povidone-iodine group began to increase on the time point of $24 \mathrm{~h}$ as well. The increased levels of these two groups didn't fluctuate much. On the time point of $48 \mathrm{~h}$, the level of IL-6 in the Sanhuang group was lower than that in the povidone-iodine group (see Figure 3).

\section{Discussion}

After severe burn, blood perfusion in renal tissues is deficient, the blood flow in the kidney is decreased and reassigned, and the blood flow in the renal cortex is obviously decreased from $80 \%$ to $10 \%$ of total RBF. When ischemia happens to the kidney, renal vascular resistance 
is increased and the glomerular capillary pressure is decreased, which leads to the decrease of glomerular filtration rate. After severe burn, a series of neutral, humoral and cellular reactions are activated in the body, and many kinds of vasoactive substances are generated, such as catecholamine, 5-hydroxytryptamine, kinin, arachidonic acid and other metabolites, some of which (lysosomal enzyme and oxygen free radical, etc.) are probably associated with renal failure caused by burn injuries. ${ }^{[1]}$ Our research results showed that Sanhuangshaoshangling could lower the level of MPO in renal tissues, reduce leukocyte infiltration locally and improve the renal function.

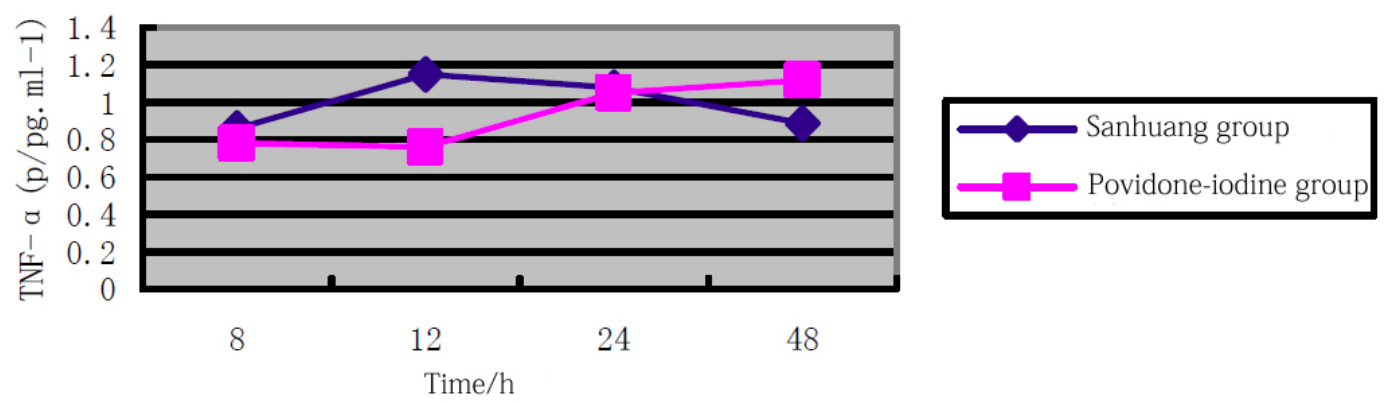

Figure 2: Changes in the level of TNF- $\alpha$ after burns

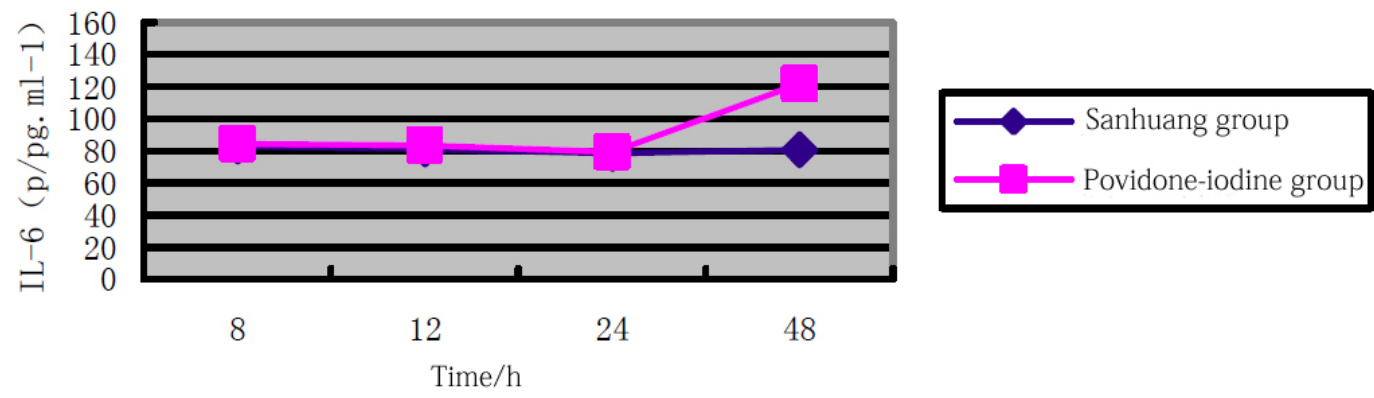

Figure 3: Changes in the level of IL-6 after burns

$\mathrm{Cr}$ and Bun are metabolic end products of proteins and creatine respectively, and can be excreted from the kidney. The levels of $\mathrm{Cr}$ and Bun in serums can be used to evaluate the renal function, but they cannot be considered as early and sensitive markers of impaired renal function. ${ }^{[2]}$ Studies have showed that, according to the science of traditional Chinese medicine, "four syndromes and four diagnostic methods" can be used as syndrome differentiation in the treatment of renal ischemia-reperfusion injury. "Blood stasis syndrome", is represented by depletion of Yin causing Yang collapse in the stage of obstruction and rejection, Qi and Yin deficiency in the stage of asthenic disease. The method of "promoting blood circulation for removing blood stasis" is one of "four methods" mentioned above, and it can be applied to the treatment of renal ischemia-reperfusion injury by use of traditional Chinese medicine with the effect of promoting blood circulation for removing blood stasis. This method is appropriate to the treatment of blood stasis syndrome with promising application prospects, as it has effects of invigorating Yin and down-bearing the turbid, restoring Yang and rescuing patients from collapse, as well as reinforcing Qi and nourishing Yin. ${ }^{[3]}$
Scutellaria baicalensis, platycladus orientalis and coptis chinensis in the ingredients of Sanhuangshaoshangling have effects of catharsis and purgation; Lonicera japonica and taraxacum mongolicum have effects of clearing heattoxicity. Sanhuangshaoshangling can inactivate the toxicity or reduce toxin by changing the structure of endotoxin after uptake into tissues. ${ }^{[4]}$ It can protect viscera by reducing the release of endotoxin, facilitating the excretion of endotoxin and weakening or eliminating the effects of TNF- $\alpha$ and IL-6. ${ }^{[5]}$

Sanhuangshaoshangling, a kind of ointment, can be used as a method to promote blood circulation for removing blood stasis and clear heat-toxicity. It can effectively protect from the invasion of bacteria and relieve wound pains as it can effectively prevent from the stimulation of the air to nerve endings. Besides, it can also improve the micro circulation and promote wound healing. Sanhuangshaoshangling can keep penetrating for a long time and remain its moist character. Therefore, it can not only improve the therapeutic effect, but also have no toxic and stimulary effects on the normal tissues. ${ }^{[6]}$ 


\section{Conflicts of Interest Disclosure}

The authors have no conflicts of interest related to this arti-

\section{References}

[1] Wang Q, Yao YM, Wang WJ, et al. Effect of Xuebijing Injection on Renal High Mobility Group Box-1 Protein Expression and Acute Kidney Injury in Rats after Scald Injury. Acta Academiae Medicinae Sinicae. 2007; 29(4): 478-483. PMid: 19209788.

[2] Liu BY, Liu BY, Li X, et al. The study of protective effect and mechanisms of lmwh on kidney injury of rats with severe acute pancreatitis. Chinese Medical Engineering. 2012; 20(1): 16-18.

[3] Zhu L. Experimental study on anti-shock effect of traditional Chinese medicine. Journal of Emergency in Traditional Chinese cle.

Medicine. 1999; 8(1): 42-43.

[4] Wang MQ, Sun YH, Li XC, et al. Concentration changes of circulating LPS, TNF- $\alpha$ and IL-6 in rats with deep thickness burns after woundabrasion in shock stage. Journal of Shandong University (Health Science). 2003; 41(4): 427-429.

[5] Xiao H, Wang DC, Leng XF, et al. Effects of early fluid resuscitation on TNF- $\alpha$, IL- 6 and IL- 8 in lymphatic circulation of burned rats. Journal of Shandong University (Health Science). 2005; 43(10): 964-967.

[6] Ma SQ. The pathological view and treating principle of burn wound of traditional Chinese medicine. The Chinese Journal of Burns Wounds and Surface Ulcers. 1998(2): 60-62. 